\title{
JUSTIFICATION OF EVALUATION CRITERIA OF GOLD BENEFICIATION ABILITY BY GRAVITATIONAL METHODS
}

\author{
Tatyana Alexandrova*, Artyem Romashev, Vadim Potemkin \\ Saint Petersburg Mining University, Saint Petersburg, Russian Federation
}

The development of science and technology involves the use of specialized software packages designed, among other things, for mathematical modeling and simulation of enrichment processes. One of the goals of using this type of softwareis to optimize technological treatment processes, design of processing plants, etc. However, since the corresponding software is not always freely available, or it does not exist, there is a need to develop application tools. The objective is to study the application of object-oriented programming to evaluate the enrichment of raw materials by gravitational methods. This paper presents the results of mathematical modeling of the gravitational separation of a mixture of polymineral gold-pyrite, gold-quartz, and gold-arsenopyrite intergrown pieces. The obtained alignment charts allow estimating the enrichment of raw materials depending on the unlocking degree ofintergrown pieces. As a result of the study, a criterion was proposed to evaluate the possibility of gravitational grain separation, grain size, and unlocking degree. A chart for evaluating the possibility of gravitational separation was proposed.

Key words: gravitational enrichment, object-oriented programming, Python, complex ores, deposition rate, enrichment criterion, mathematical modeling

\section{INTRODUCTION}

Increased production of non-ferrous, rare and precious metals is accompanied by an increase in the production of complex minerals, the involvement in the operation of new fields characterized by a relatively low content of valuable components in raw materials, the vast development of progressive enrichment methods, the introduction of new machines and devices into production. The increase in the production of many minerals was made possible by developing, among others, gravitational methods of enrichment. Currently, the share of gravity methods in the processing of oxidized iron, complex, and auriferous ores is increasing every year [1]. Equipment of modern gravity mills with highly productive machine makes it possibleto simplify the machine flow sheet, ensure more economical use of production area and premises, resulting in lower capital costs ofconstruction, reduce the number of staff, increase productivity, and reduce processing costs. For processing complex raw materials, concentration table enrichment [2], centrifugal concentrators [3], and heavy-medium enrichment [4] are most often used.

Gravitational enrichment processes in the range of the original characteristics of enriched raw materials, a variety of conditions of their application in the flow sheets of processing plants, the ease of the manufacturing complex, and the use of high-performance processing machines in comparable conditions are superior to many other processes of enrichment and ensure sufficient separation of mineral mixtures with relatively low material, labor, and energy costs [1]. The possibility of using gravitational processes is characterized by differences in the density, size, and shape of the intergrown pieces, as well as the unlocking degree, since the percentage of useful mineral content in each particular intergrown piece depends on the unlocking degree.

More than ten graphical and analytical methods have been proposed as an enrichment criterion. Graphical methods are based on the distribution curves of fraction density, slope and deflection angles, etc. Analytical methods use the product yield of a given density, the fraction yield of intermediate density, and so on as an enrichment criterion [5].

In practice, enrichment is characterized by the content in a mixture of minerals of intermediate density $( \pm 100$ $\mathrm{kg} / \mathrm{m}^{3}$ ) to separation density as small oscillations of the latter cause mutual clogging of separation products. According to this classification, when the content of the intermediate density fractions is 0 to $7 \%$, the ore is considered free-milling; from 7 to 10 - medium; from 10 to 15 - refractory; 15 to 20 - very hard; from 20 to 25 - extremely hard and more than 25 - super-hard [5].

A criterion can be chosen that characterizes the ratio ofdensities of the separated minerals to evaluate the possibility of using gravitational enrichment [5]:

$K=\frac{\rho_{\mathrm{M} 1}-\rho_{\mathrm{A}}}{\rho_{\mathrm{M} 2}-\rho_{\mathrm{A}}}$

where $\rho_{M 1}$ and $\rho_{M 2}$ are densities of the studied minerals $\left(\mathrm{g} / \mathrm{cm}^{3} \rho_{A}\right.$ - medium density $\left(\mathrm{g} / \mathrm{cm}^{3}\right)$

It is known that when this ratio is greater than 2.5 , a mixture of minerals with density $p_{M 1}$ and $p_{M 2}$ is free-milling and their separation is possible in case the particle size is up to the size of fine sand; ata ratio equal to 1.75 , separation in an industrial environment is free-milling if the particle size down to 100 mesh $(0.140 \mathrm{~mm})$; when the ratio is 1.5 , the separation is difficult, and the lower limit 
of particle size for industrial conditions is 10 mesh (1.6 $\mathrm{mm}$ ); at a ratio of 1.25 ,separation is possible for sizes of gravel and less than 1.25 gravity separation is almost impossible. However, for each specific raw material, these indicators may be different. This criterion does not take into account the particle size, which can lead to incorrect result interpretation. For example, it was found that an intergrown piece with a density of $15840 \mathrm{~kg} / \mathrm{m}^{3}$ and a size of $480 \mu \mathrm{m}$ hada final subsidence rate close to a piece with a density of $6238 \mathrm{~kg} / \mathrm{m}^{3}$ and a size of $840 \mu \mathrm{m}$ (with the same shape and subsidence conditions), withthe enrichment ratio $\mathrm{K}=2.53$. In connection with the above, it is necessary to develop a fundamentally different criterion that would take into account the particle size, i.e., based on the fall velocity.

For all gravitational processes, there is a fall of particles in a liquid or gas. The distance traversed by particles in the vertical direction is measured in tens of centimeters and meters in some gravity devices (in hydraulic classifiers, concentrators), in others in millimeters (in spiral separators, stoppers). Regularities of falling particles in a liquid or gas are essential for creating a theory of gravitational processes and for calculating machines and mechanisms. In gravitational devices, particles of various sizes, densities, and shapes fall together in the liquid. Each of these parameters affects the particle fall velocity. The mutual influence of particles is also significant, especially noticeable at high concentrations. Meanwhile, it was found that at the output of the chopping cutter (especially at large aggregate volumes), different size classes hada similar shape (and as a consequence, the shape ratio), so the influence of the shape in the criterion output can be neglected $[6,7]$. Mill drain usually contains intergrown pieces in the entire range of their unlocking (from $\rho_{\max }$ to $\rho_{\min }$ ) and size, then the selectivity ratio for unlocking intergrown pieces $\varphi$ can be calculated from the expression:

$$
\varphi=\sqrt{(50-\lambda)^{2}} \text {, }
$$

here $\lambda$ is the volume content of a mineral with a density of $p_{\max }$ or $p_{\min }$.

Following this indicator, the full mineral release is achieved in the absence of other impurities, i.e., if $\lambda=100$ then the selectivity ratio $\varphi=100 \%$, similarly at $\lambda=0$ (i.e., in the intergrown piece the content of one of the specific heavy and specific light components is 0$) \varphi=100 \%$.
The second proof of the applicability of this criterion is that for an equal volume distribution of the specific heavy and specific light mineral in the sample $(\lambda=0.5)$, the selectivity ratio is $\varphi=0 \%$. Based on the above, two cases are possible for an array of intergrown pieces, when the selectivity ratio values are equal depending on the unlocking degree and, therefore, a pair can be formed from the calculated intergrown piece fall velocity of equivalent unlocking degrees. The greater the relative difference between these values, the more likely it is to perform the process of separation according to the fall velocities.

$$
\Delta v=v_{\varphi}^{\rho_{\max }}-v_{\varphi}^{\rho_{\min }}
$$

where $v_{\varphi}^{\rho_{\max }}$ and $v_{\varphi}^{\rho_{\min }}$ are the fall velocities of intergrown pieces with the predominance of the specific light and specific heavy mineral, respectively, at the value of the selectivity ratio $\varphi ; p_{\max }$ and $p_{\min }$ - the densities of the specific heavy and specific light mineral in the ore.

In this paper, a simulation exercise was performed to calculate the rates of constrained deposition of polymineral intergrown pieces with a size of $-1+0.040 \mathrm{~mm}$, such as:

- $\quad$ Gold + Quartz $\left(\mathrm{Au}+\mathrm{SiO}_{2}\right)$

- Pyrite + Quartz $\left(\mathrm{FeS}_{2}+\mathrm{SiO}_{2}\right)$

- Arsenopyrite + Quartz $\left(\mathrm{FeAsS}+\mathrm{SiO}_{2}\right)$

- The feasibility of the study is determined by the following factors:

- Often native gold is found as small and oval inclusions in pyrite (Figure 1).

- Also, native gold can be observed in gravitational concentrates in fusion with arsenopyrite [8].

- Gravity enrichment is one of the primary stages of processing gold sulfide ores [9].

\section{MATERIALS AND METHODS}

The study of the separation processes of heterogeneous systems, as well as the interaction of the constituent phases, involves solving the problem of calculating the velocities of bodies in the dispersed phase. There are various methods for estimating the final fall velocity of mineral grains [10]:

- by the oretical equations;

- using empirical and interpolation formulas;

- using graphs and tables based on experimental data.

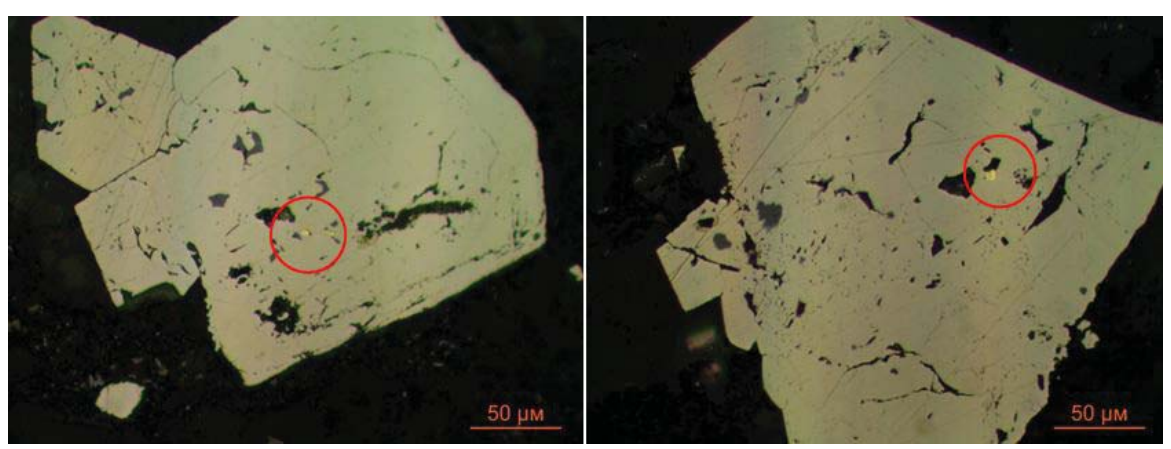

Figure 1: Crystallization of native gold in pyrite 
In practice, the most frequently used theoretical formulas have applicability conditioned by the limitation on the size of the studied particles. Many different formulas for calculating the free-fall velocity of spherical particles are given in the literature, but at the same time the question of the accuracy of the data obtained remains open. Comparative analyses of formulas for particle free fall velocities are given in many papers [1, 2, 6, 11-14]. However, there are many new publications in the press devoted to this issue. In practice, the most commonly used formulas for the free-fall velocity are the formulas of Stokes, Allen, and Rittinger.

Stokes'formula is applicable for laminar motion within $\mathrm{Re}$ up to 0.5:

$v=\frac{g\left(\rho_{\mathrm{s}}-\rho_{1}\right) d^{2}}{18 \mu}$

where $p_{s}$ and $p_{1}$ are the density of the solid phase and the liquid, respectively; $\mathrm{g}$ - free-fall acceleration, $\mathrm{m} / \mathrm{s}$; $\mu$ - dynamic viscosity, $v-$ fall velocity, $\mathrm{m} / \mathrm{s} ; d$ - particle diameter, $\mathrm{m}$.

For particles of intermediate size $(1<R e<1,000)$, the formula was experimentally established by Allen:

$v=K_{A} \frac{d}{\sqrt[3]{v}} \sqrt[3]{\left(\frac{\rho_{\mathrm{s}}-\rho_{\mathrm{l}}}{\rho_{\mathrm{l}}}\right)^{2}}$,

where $K_{A}$ is the Allen coefficient, which is equal to 24.3 or 25.8 according to [11] for spherical-shaped particles;y the kinematic ratio of the medium viscosity, $\mathrm{cm}^{2} / \mathrm{s}$.

For a region of turbulent motion (Re from 1,000), Rittinger's formula shows good convergence:

$v=K_{R} \sqrt{\left(\frac{\rho_{\mathrm{s}}-\rho_{1}}{\rho_{1}}\right) d}$,

where $K_{R}$ is the Rittinger ratio, which is equal to 5.53 or 4.43 according to [11] for spherical particles.

Stokes', Allen's, and Rittinger's formulas have relatively low square deviations compared to the experimentally obtained data, but can only be used in a limited range of Reynolds numbers and do not take into account the constrained nature of deposition, as well as the size of the studied particle class $(-1+0.040 \mathrm{~mm})$.

Professor Lyashchenko developed a universal method that is used to calculate the deposition rate of bodies of any size, density, and shape [5].

The essence of the method is that for known grain and medium parameters, the dimensionless parameter $\operatorname{Re}_{2} \Psi$ is calculated using the formula (2):

$\operatorname{Re}_{2} \Psi=\frac{\pi \cdot d^{3}}{6} \cdot(\delta-\Delta) \cdot g \cdot \frac{\Delta}{\mu^{*}}$,

where $\mathrm{d}$ - the particle diameter, $\mathrm{mm} ; \delta$ - the particle density $\mathrm{kg} / \mathrm{m}^{3} ; \Delta-$ the medium density, $\mathrm{kg} / \mathrm{m}^{3}, \mu$ - the medium viscosity, $\mathrm{n} \cdot \mathrm{s} / \mathrm{m}^{2}$.

According to the value $\operatorname{Re}_{2} \Psi$, the corresponding value of the Reynolds parameter is determined by the graphical analytic method using the diagram, and then the particle velocity is calculated using the formula (3) [5]:

$v_{0}=\frac{R e \cdot \mu}{d \cdot \rho_{f}}$.

As is known, the velocity of constrained fall is lower than the velocity of the free fall, so for a more accurate assessment, it is necessary to introduce a correction ratio for the velocity reduction:

$v_{C T}=\theta \cdot v_{0}$,

where the value of the coefficient $\theta$ is usually in the range of $0.08-0.21$, but can be calculated from the expression:

$\theta=0.6 d^{0.23} \frac{\rho}{2.65}$

Despite the advantages of the chosen method for estimating the final fall velocity of polymineral intergrown pieces, one of the main problems that cause the complexity of calculations is the need to calculate these velocities for a large number of intergrown variants. In order to solve the problem, object-oriented programming methods were used.

Object-oriented programming is a methodology where a program is a collection of objects-instances of classes that form an inheritance hierarchy. In this case, the approach to programming (modeling of information objects) solves the following issues:

- $\quad$ structuring, facilitating the modeling process;

- minimizing data redundancy.

In the case of solving the problem, the tools of high-level programming languages were used, in particular, Python with the integration of calculated data into the Microsoft Excel package [15;16-19] .

At the initial stage of evaluating the final fall velocity of polymineral grains, the software developed at the Department of Mineral Enrichment of St. Petersburg Mining University was used (language: Delphi 7, Reg. No. 2019612243 from February 13, 2019), implementing the method described above (Figure 2).

The program is designed to calculate the deposition rate of mineral particles of different sizes using Lyashchenko's method described above. The following characteristics are provided as input data for calculation: names and densities of particles, their size, pulp density, the substance proportion in the suspension, as well as the deposition medium. The functionality of the program allows saving the calculated data in a file with the .xls extension; thename is generated automatically.

However, considering the specifics of the problem under consideration, the functionality of the developed program was significantly expanded by means of the Python language. The peculiarity of this language, as well as the reason for its use, are the following: convenience of processing large data sets, clear and simple syntax, cross-platform compatibility, a huge number of services, development environments and frameworks, the ability to connect libraries written in $\mathrm{C}$, dynamic typing, and program execution speeds. 


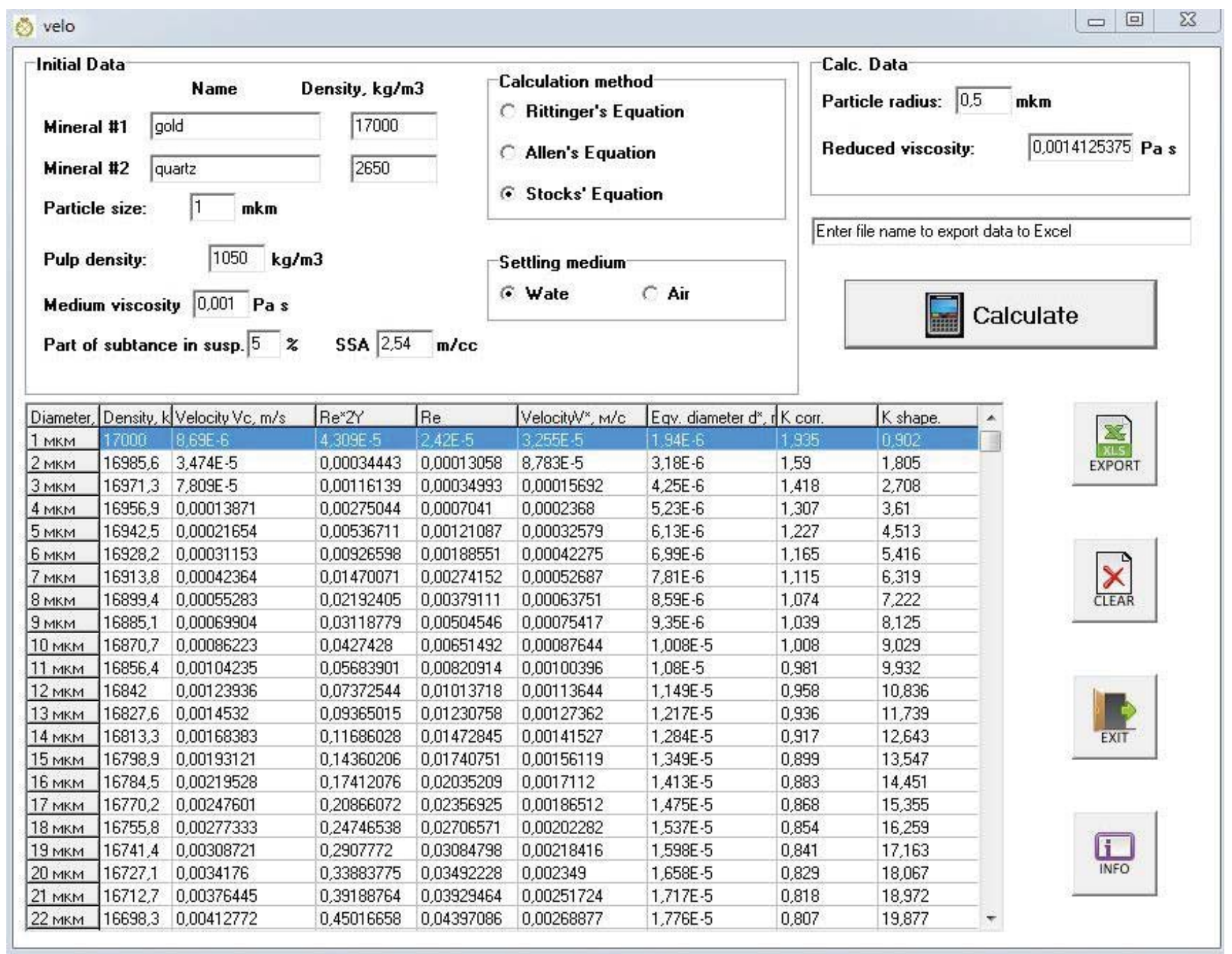

Figure 2: Program for deposition rate calculation

Using a specialized Python library for processing large Num Pyarrays, as well as a previously developed Delphi program, primary data were obtained (Table 1) and the values of $\Delta v$ were calculated:

\section{DISCUSSION AND RESULTS}

The "velocity field" was calculated for all possible types of intergrown pieces with a uniform distribution of densities between monofractions from $p_{\max }$ to $p_{\min }$ and a size from 1 to $1,000 \mu \mathrm{m}$. The array of the studied velocity field was $10^{6}$ values, which provides a search of all possible options for the unlocking degree of intergrown pieces of the polymineral mixture and, importantly, the calculation accuracy.
In order to reduce the size of the velocity field visualization and make it easier to work with data, the particles were clustered into groups with similar fall velocity and size, resulting in generalized data arrays of smaller size $-25 \times 25$ values (Figure 3 ).

Also, to account for the effect of viscosity for each of the three types of intergrown pieces, a simulation exercise was performed at different solid content $(5,25,50 \%)$. Thus, 9 fields were obtained for visual evaluation of the distribution of the final deposition rates of polymineral intergrown pieces, depending on the density, size and viscosity of the suspension. Recoding the color scheme of the velocity fields shown in (Figure 4) allows drawing the following conclusion about the relative distribution of velocities between all nine simulation exercises.

Table 1: General view of the "velocity fields" array

\begin{tabular}{|c|c|c|c|}
\hline Intergrown pieces size, $\mu \mathrm{m}$ & \multicolumn{3}{|c|}{ Intergrown pieces density, $\mathrm{kg} / \mathrm{m}^{3}$} \\
\hline 1 & $\rho_{1}$ & $* * *$ & $\rho_{2}$ \\
\hline$* * *$ & $\mathrm{~V}_{1}$ & $* * *$ & $* * *$ \\
\hline$* * *$ & $* * *$ & $* * *$ & $* * *$ \\
\hline 1,000 & $* * *$ & $* * *$ & $\mathrm{~V}_{10}{ }^{6}$ \\
\hline
\end{tabular}


selectivity coefficient ( $5 \%$ of solid)

\begin{tabular}{|c|c|c|c|c|c|c|c|c|c|c|c|c|}
\hline dMv & 100 & 92 & 83 & 75 & 67 & 58 & 50 & 42 & 33 & 25 & 17 & 8 \\
\hline 40 & 0,005 & 0,005 & 0,004 & 0,004 & 0,004 & 0,003 & 0,003 & 0,002 & 0,002 & 0,001 & 0,001 & 0,000 \\
\hline 80 & 0,015 & 0,013 & 0,012 & 0,011 & 0,010 & 0,008 & 0,007 & 0,006 & 0,005 & 0,004 & 0,002 & 0,001 \\
\hline 120 & 0,026 & 0,024 & 0,022 & 0,019 & 0,017 & 0,015 & 0,013 & 0,011 & 0,008 & 0,006 & 0,004 & 0,002 \\
\hline 160 & 0,040 & 0,036 & 0,033 & 0,029 & 0,026 & 0,023 & 0,019 & 0,016 & 0,013 & 0,010 & 0,006 & 0,003 \\
\hline 200 & 0,055 & 0,050 & 0,045 & 0,040 & 0,036 & 0,031 & 0,027 & 0,022 & 0,018 & 0,013 & 0,009 & 0,004 \\
\hline 240 & 0,071 & 0,065 & 0,058 & 0,052 & 0,046 & 0,040 & 0,034 & 0,029 & 0,023 & 0,017 & 0,011 & 0,006 \\
\hline 280 & 0,088 & 0,080 & 0,073 & 0,065 & 0,058 & 0,050 & 0,043 & 0,036 & 0,029 & 0,021 & 0,014 & 0,007 \\
\hline 320 & 0,107 & 0,097 & 0,088 & 0,079 & 0,070 & 0,061 & 0,052 & 0,043 & 0,035 & 0,026 & 0,017 & 0,009 \\
\hline 360 & 0,127 & 0,115 & 0,104 & 0,093 & 0,083 & 0,072 & 0,062 & 0,051 & 0,041 & 0,031 & 0,020 & 0,010 \\
\hline 400 & 0,147 & 0,134 & 0,121 & 0,109 & 0,096 & 0,084 & 0,072 & 0,060 & 0,048 & 0,036 & 0,024 & 0,012 \\
\hline 440 & 0,169 & 0,154 & 0,139 & 0,124 & 0,110 & 0,096 & 0,082 & 0,068 & 0,055 & 0,041 & 0,027 & 0,014 \\
\hline 480 & 0,191 & 0,174 & 0,157 & 0,141 & 0,125 & 0,109 & 0,093 & 0,077 & 0,062 & 0,046 & 0,031 & 0,015 \\
\hline 520 & 0,215 & 0,195 & 0,177 & 0,158 & 0,140 & 0,122 & 0,104 & 0,087 & 0,069 & 0,052 & 0,035 & 0,017 \\
\hline 560 & 0,239 & 0,217 & 0,196 & 0,176 & 0,156 & 0,136 & 0,116 & 0,097 & 0,077 & 0,058 & 0,038 & 0,019 \\
\hline 600 & 0,263 & 0,240 & 0,217 & 0,194 & 0,172 & 0,150 & 0,128 & 0,107 & 0,085 & 0,064 & 0,042 & 0,021 \\
\hline 640 & 0,289 & 0,263 & 0,238 & 0,213 & 0,189 & 0,164 & 0,141 & 0,117 & 0,093 & 0,070 & 0,047 & 0,023 \\
\hline 680 & 0,315 & 0,287 & 0,259 & 0,232 & 0,206 & 0,179 & 0,153 & 0,128 & 0,102 & 0,076 & 0,051 & 0,025 \\
\hline 720 & 0,342 & 0,311 & 0,281 & 0,252 & 0,223 & 0,195 & 0,166 & 0,138 & 0,111 & 0,083 & 0,055 & 0,028 \\
\hline 760 & 0,370 & 0,336 & 0,304 & 0,272 & 0,241 & 0,210 & 0,180 & 0,150 & 0,119 & 0,089 & 0,060 & 0,030 \\
\hline 800 & 0,398 & 0,362 & 0,327 & 0,293 & 0,260 & 0,226 & 0,194 & 0,161 & 0,129 & 0,096 & 0,064 & 0,032 \\
\hline 840 & 0,427 & 0,388 & 0,351 & 0,314 & 0,278 & 0,243 & 0,208 & 0,173 & 0,138 & 0,103 & 0,069 & 0,034 \\
\hline 880 & 0,456 & 0,415 & 0,375 & 0,336 & 0,298 & 0,260 & 0,222 & 0,185 & 0,147 & 0,110 & 0,074 & 0,037 \\
\hline 920 & 0,486 & 0,442 & 0,400 & 0,358 & 0,317 & 0,277 & 0,237 & 0,197 & 0,157 & 0,118 & 0,078 & 0,039 \\
\hline 960 & 0,517 & 0,470 & 0,425 & 0,381 & 0,337 & 0,294 & 0,251 & 0,209 & 0,167 & 0,125 & 0,083 & 0,042 \\
\hline 1000 & 0,548 & 0,499 & 0,451 & 0,404 & 0,357 & 0,312 & 0,267 & 0,222 & 0,177 & 0,133 & 0,088 & 0,044 \\
\hline
\end{tabular}

Figure 3: Example of clustering of the $\mathrm{Au}+\mathrm{SiO}_{2}$ deposition rate field (5\% solid), where the upper-right corner corresponds to the value for a monomineral quartz particle with a size of $1 \mu \mathrm{m}$, the lower-left corner corresponds to a monomineral gold particle with a size of $1000 \mu \mathrm{m}$

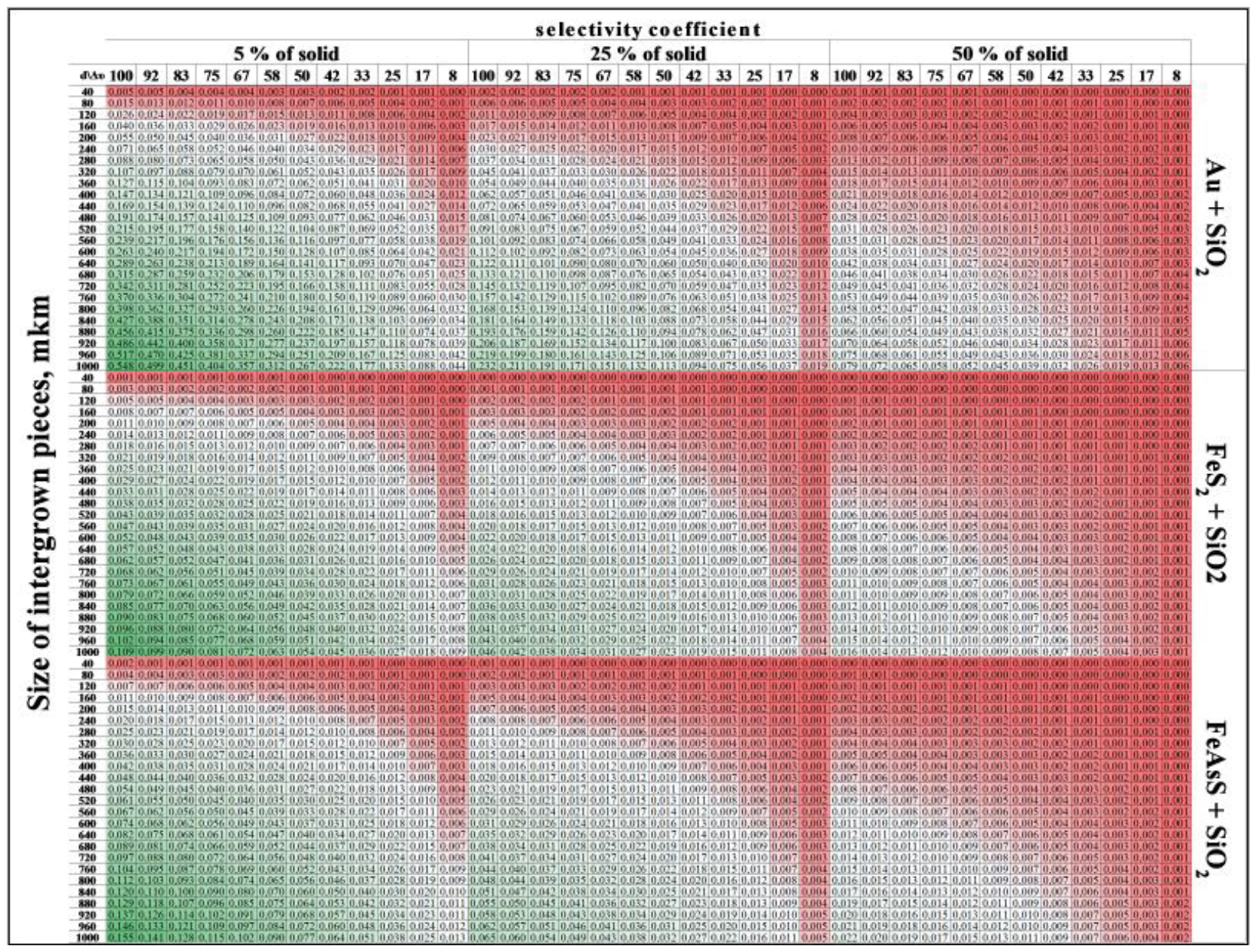

Figure 4: The relative distribution of the final deposition rates 
As a result of the conducted simulation exercise, it was found that in the mode of constrained deposition, the concentration of solid in the suspension, together with the difference in the densities of the studied minerals, had a significant impact on the nature of the velocity distribution. The smaller the density difference, the greater the effect of viscosity on the separation process. Data visualization also allowed identifying three characteristic zones for differences in the fall velocity. It is characteristic that these regions do not depend on minerals and have similar forms. Then the following criterion can be introduced for grading by gravitational enrichment:

$K_{G}=\frac{\Delta v}{v_{100}^{\rho_{\max }}} 100$

where $v_{100}^{\rho_{\max }}-$ fall velocity of a specific heavy mineral at the selectivity coefficient $\varphi=100 \%$.
Also, using the developed software, alignment charts were obtained that reflect the nature of the distribution of particle velocities depending on the size and density of intergrown pieces. According to these charts, it is possible to determine the areas of enrichment of raw materials, taking into account the content of the useful mineral in the intergrown piece, in other words, by the unlocking degree of intergrown pieces (Figure 5). Alignment charts have an identical appearance for all three types of intergrown pieces considered and can be zoned by the developed scale of gravitational enrichment, the values of which are based on the calculated criteria (Figure 5).

\section{CONCLUSION}

As a result of the performed research, the final fall velocity of three types of the most typical for auriferous ores intergrown pieces was investigated. A brief review

selectivity coefficient

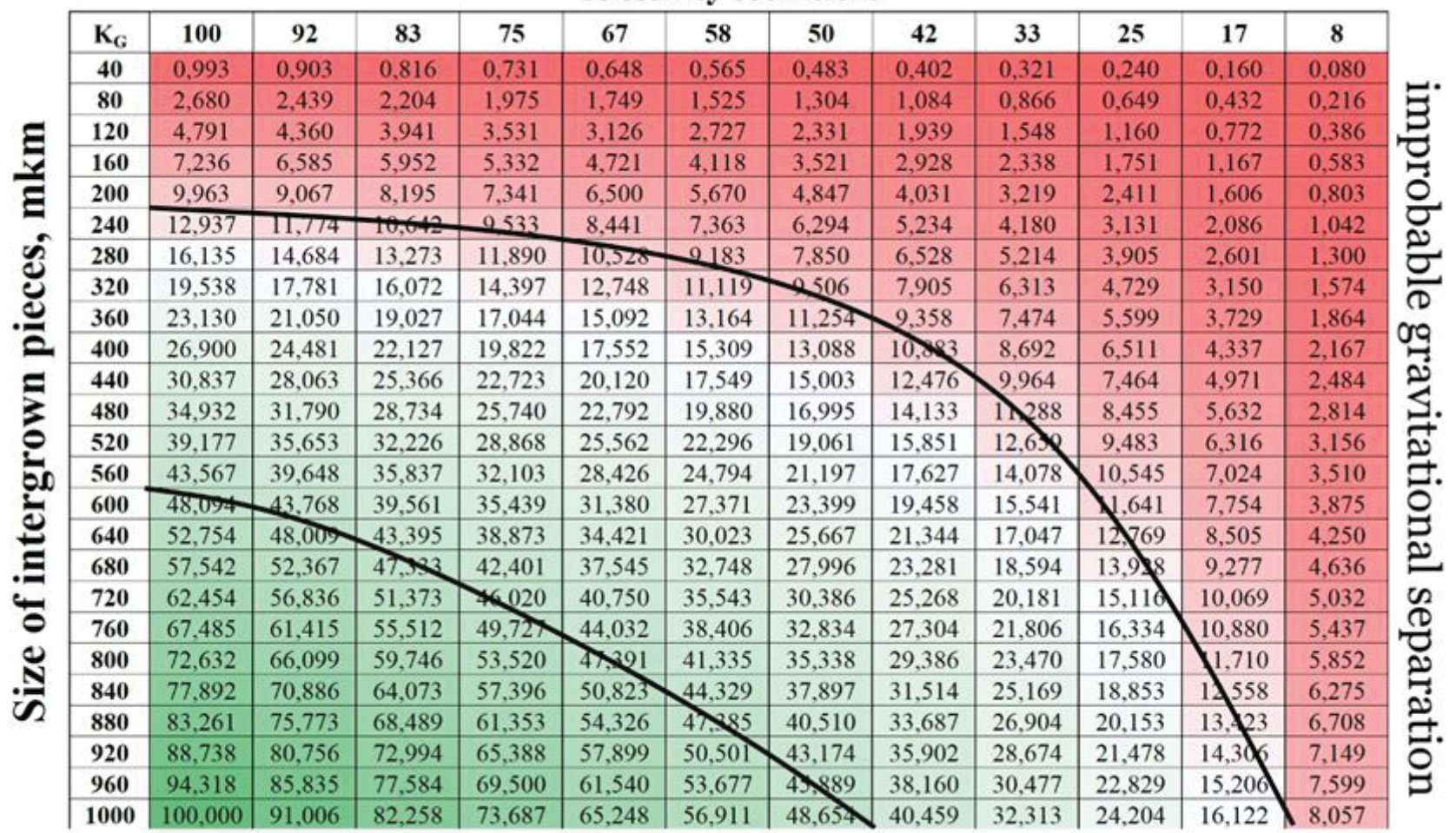

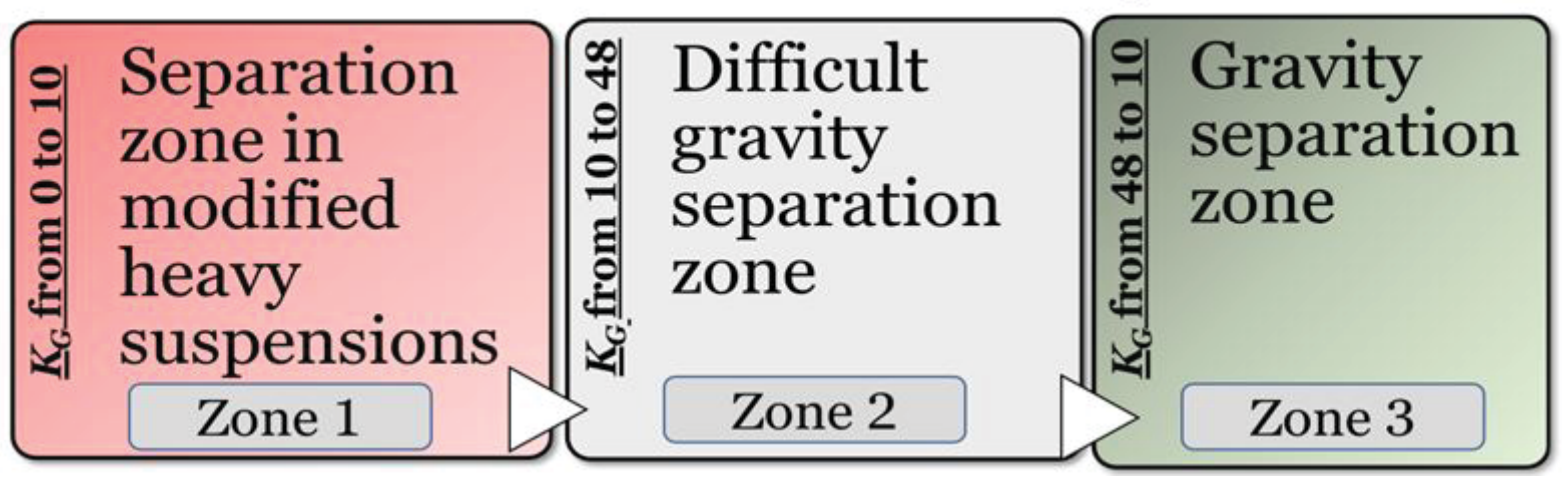

Figure 5: Enrichment criterion chart for gold-quartz intergrown pieces 
of the developed methods for calculating velocities was carried out, and the most convenient method was chosen - Lyashchenko's method, which is the basis of the developed software. In addition to direct calculations, the program allows calculating the so-called "velocity field" - an array of all possible options within a given size and the entire unlocking range (based on the assessment of the unlocking degree by the proposed selectivity coefficient). The calculations made it possible to obtain alignment charts to determine the probability with which particles of a specific size and density (intergrown pieces with a certain ratio (for example, gold - quartz) are separated using gravitational devices. Processing of the obtained charts allowed justifying the criterion of gravitational enrichment, which can be used in the development of projects for new factories and the study of ores for the possibility of gravitational enrichment.

\section{ACKNOWLEDGMENTS}

The work is carried out with financial support of Ministry of Education and Science of Russian Federation according to grant program "Research and development in priority areas of development of Russia's scientific and technical complex for 2014 - 2020", the project № 05.616.21.0127 (RFMEFI61620X0127).

\section{REFERENCES}

1. Korchevenkov S., \& Aleksandrova T. (2018). Investigation of the influence a morphologic characteristic of the noble metal particles on gravity efficiency devices. International Multidisciplinary Scientific GeoConference: SGEM: Surveying Geology \& mining Ecology Management. Vol. 18. - Pp. 99-104

2. Myazin V.P., \& Litvintsev S.A. (2018). Increasing the efficiency of gravity extraction of gold from complex gold-polymetallic ores. Proceedings of the Siberian Branch of the Section of Earth Sciences of the Russian Academy of Natural Sciences. Geology, exploration and development of mineral deposits. Vol. 41. - No. 1 (62).

3. Algebraistova, N.K., Samorodskiy, P.N., Kolotushkin, D.M., \& Prokopyev, I.V. (2018). Technology of gold recovery from gold-bearing technogenicraw materials. Obogashchenie Rud, (1), pp. 33-37.

4. Mkandawire, N.P., Mc Grath, T., Bax, A., Eksteen, J. Potential of the dense media cyclone for gold ore preconcentration (2020) Mineral Processing and Extractive Metallurgy: Transactions of the Institute of Mining and Metallurgy, 129 (1), pp. 87-95

5. Verkhoturov M.V. (2006). Gravity methods of enrichment. Text book. for a university. M .: MAKS Press. Pp. 352.
6. Lvov V., Sishchuk J., \& Chitalov L. (2017). Intensification of Bond ball mill work index test through various methods. International Multidisciplinary Scientific Geo Conference: SGEM: Surveying Geology \& mining Ecology Management. Vol. 17. - №. 1.1. - Pp. 857-863.

7. Nikolaeva, N.V., Taranov, V.A., \& Afanasova, A.V. (2015). Ore strength analysis in planning ore pretreatment circuit Information about author. Gornyi Zhurnal, 2015 (12), pp. 9-13.

8. Algebraistova, N.K., Makshanin, A.V., Burdakova, E.A., Samorodskiy, P.N., \& Markova, A.S. (2015). Development of stage gravity flowsheet for recovery of noble metals, Obogashchenie Rud, (2), pp. 3-7.

9. Wills B. A., \&Finch J. (2019). Wills' mineral processing technology: an introduction to the practical aspects of ore treatment and mineral recovery. - Butterworth-Heinemann, 2015.

10. Wang, X., Qin, W., Jiao, F., Yang, C., Cui, Y., Li, W., Zhang, Z., Song, H. Mineralogy and pretreatment of a refractory gold deposit in Zambia (2019) Minerals, 9 (7), № 406, .

11. Lowes, Callan \& Zhou, J \& Galvin, Kevin. (2018). Gravity Separation of Gold Ore using the REFLUX ${ }^{\mathrm{TM}}$ Classifier to Achieve Coarse Particle Gangue Rejection. Chemeca. Pp. 74.

12. Vasiliev A. M. (2011). Analysis of formulas for the free fall velocity of spherical particles. Ore dressing. No. 2. - Pp. 22-26.

13. Matveev, I.A., Matveev, A.I., Eremeeva, N.G., \&Grigorev, Y.M. (2018). Experimental and theoretical investigation of the behavior of particles in water flow. Mining Informational and Analytical Bulletin, (11), pp. 171-177.

14. Aleksandrova T.N. \& Kuskov V.B. (2014).Development of methods for increasing efficiency of gravitational extraction of small and thin gold from scales of different genesis. Journal of Mining Institute. Vol. 210, p. 69-77.

15. Yemelyanov, V., Nedelkin, A., \& Yemelyanova, N. (2020). Expert system software for assessing the technical condition of critical lined equipment doi:10.1007/978-3-030-37916-2_92 
16. Stival, M., Cunha, E. J. N. S., Cunha, M., \& Rocha, J. R. C. D. (2019). Application of ludic activity for learning of chemical concepts: Case study. [Aplicacao de atividade ludica para o aprendizado de conceitos quimicos: Estudo de caso] Periodico Tche Quimica, 16(32), 783-793.

17. Yemelyanov, V. A., Yemelyanova, N. Y., Nedelkin, A. A., Glebov, N. B., \& Tyapkin, D. A. (2019). Information system to determine the transported liquid iron weight. Paper presented at the Proceedings of the 2019 IEEE Conference of Russian Young Researchers in Electrical and Electronic Engineering, El Con Rus 2019, 377-380. doi:10.1109/EIConRus.2019.8656693
18. Teles, A. C., Freitas, A. C. D. P., \& Rodrigues, A. C. (2019). Failures detection methods in chemical process using artificial intelligence. [Comparacao de metodos de deteccao de falhas em processos quimicos utilizando inteligencia artificial] Periodico Tche Quimica, 16(32), 61-68.

19. Yemelyanov, V. A., Nedelkin, A. A., \& Olenev, L. A. (2019). An object-oriented design of expert system software for evaluating the maintenance of lined equipment. Paper presented at the 2019 International Multi-Conference on Industrial Engineering and Modern Technologies, Far East Con 2019, doi:10.1109/FarEastCon.2019.8934414 